\title{
FLOW GENERATED DUE TO LONGITUDINAL AND TORSIONAL OSCILLATIONS OF A CIRCULAR CYLINDER WITH SUCTION/INJECTION IN A MICROPOLAR FLUID
}

\author{
J. V. RAMANA MURTHY, G. NAGARAJU AND P. MUTHU
}

\begin{abstract}
The flow generated by performing longitudinal and torsional oscillations of a porous circular cylinder which is subjected to constant suction/injection at the surface of the porous cylinder is studied. A finite difference method is proposed to analyse the velocity components and micro-rotation components, in an infinite expanse of an incompressible micropolar fluid. The effects of cross viscosity parameter, couple stress parameter, Reynolds number and Gyration parameter on the axial and torsional velocity components and on the micro-rotation components are shown graphically. Drag force acting on the wall of the cylinder is derived and the effects of micropolar parameters and suction parameter on the drag are shown graphically.
\end{abstract}

\section{Introduction}

The flow and heat transfer of certain fluids such as animal blood, real fluids with suspensions, colloidal fluids and polymer solutions, could not be explained successfully on the basis of Newtonian and non-Newtonian fluid flow theory. With this in mind, Eringen $[1,2]$ developed the theory of new class of fluids called as micro-fluids. This theory deals with a class of fluids, which exhibit certain microscopic effects arising from the local structure and micromotions of the fluid elements. These fluids, even in the simplest case of constitutively linear theory, contain 22 viscosity and material coefficients with a system of 19 partial differential equations with 19 unknowns and the solving of these equations is highly difficult.

Hence Eringen [3] latter considered a subclass of these fluids called as micropolar fluids. In these fluids the gyration tensor is the skew-symmetric, in addition to a condition of micro isotropy, so that the system of 19 equations now reduces to seven equations in seven unknowns only. This theory of micropolar fluids is the most generalization of viscous fluid

Corresponding author: J. V. Ramana Murthy.

2010 Mathematics Subject Classification. Primary 421305, 42B08.

Key words and phrases. Micropolar fluid, longitudinal and torsional oscillations, drag, micro-rotation, suction and injection. 
theory. A number of micro-continuum theories have been developed for describing the peculiar behavior of fluids containing substructures, which can translate, rotate, or even deform independently $[4,5]$.

The motion of fluids through porous permeable surfaces at low Reynolds numbers has been an important subject in the field of chemical, biomedical, and environmental engineering and science. This phenomenon is fundamental in nature and is of great practical importance in many diverse applications like production of oil and gas from geological structures, the gasification of coal, the retorting of shale oil, filtration, surface catalysis of chemical reactions, adsorption, coalescence, drying, ion exchange and chromatography.

Starting from Couette flows, the flow generated in fluids by the motion of surfaces have been attracting the researchers. Among them, the study of flow longitudinal and torsional oscillations presents some interest in different engineering areas like Oceanography, the technology of vibrations on machinery, the process of certain polymer liquid crystals, and the offshore drilling of oil. There are three physical situations in which the study of the longitudinal and torsional oscillations of the flow of micropolar fluid can be applied. The first application is in lubrication theory. The cylindrical bearings containing a non ewtonian fluid lubricant are subject to longitudinal and torsional vibrations on the machinery. A second application is the flow of polymer liquid crystals made of dumbbell like molecules processed inside a circular cylinder which is subject to longitudinal and torsional oscillations. And finally, a possible third application is the flow of mud in the drill string of an offshore oil drilling unit which is subjected to oscillations due to oceanic waves [6].

The motion of a classical viscous fluid due to the rotation of an infinite cylindrical rod immersed in the fluid was first described by stokes [7]. Later many flow problems due to the motion of bodies were solved. Some flow problems related to the motion of a cylindrical rod performing longitudinal and torsional oscillations are given below. Casarella et al.[8] studied the external flow due to longitudinal and torsional oscillations of a rod in a Newtonian fluid and obtained an exact solution. Rajagopal [9] studied the same problem for the case of a second grade fluid. Ramkissoon et al.[10] studied the internal flow due to longitudinal and torsional oscillations of a viscous fluid and they derived an analytical expression for velocities, shear stresses and drag on the cylinder. Majumdar et al.[11] obtained an exact solution for an infinite rod undergoing both longitudinal and torsional oscillations in a polar fluid and they have presented the effect of micropolar parameters on the microrotation and velocity fields graphically. Calmelet-Eluhu et al.[6] studied the internal flow of a micropolar fluid inside a circular cylinder subject to longitudinal and torsional oscillations and they have shown the effect of micropolar fluid on two components velocity field through graphs. Owen and Rahman [12] studied the same type of flow with an Oldroyd-B liquid. The problem of steady flow in a straight channel (between two parallel plates) with suction velocity at the porous walls 
was initiated by Berman [13]. Since then many problems were considered with suction velocity at the porous surfaces in different geometries and with varieties of fluid models. Kapur [14] has studied the steady flow of a non-Newtonian fluid in an annulus between two coaxial rotating cylinders with uniform angular velocities, when there is suction at one wall and injection at the other. Jangi Lal [15] studied the unsteady, viscous incompressible fluid flow around a porous infinite circular cylinder oscillating harmonically with constant suction and he solved the energy equation for the given large and small values of time. He has shown graphically the steady part of the velocity with suction and without suction case. Pontrelli [16] has studied the axi-symmetric flow of Oldroyd-B fluid due to longitudinal and torsional oscillations with suction or injection velocity applied at the surface and he has shown the effects on the non-Newtonian parameters on velocity and shear stresses through graphs. Emin Erdogan et al.[17] obtained the analytical solution in terms of confluent hyper geometric functions for the flow of a second-grade fluid between two coaxial cylinders with porous walls and he has shown the effect of moment-coefficient on Reynolds number, the elastic number and the ratio of the radii of the cylinders through graphs. Rao et at.[18] studied the unsteady viscous flow around transversely and longitudinally oscillating circular cylinder by using finite difference simulation method and they have presented flow fields graphically for a sub-critical Reynolds number of order $4 \times 10^{3}$ and $4 \times 10^{4}$. Pontrelli et al.[19] studied the steady flow of an OldroydB fluid between two porous concentric circular cylinders. They found velocity by solving a system of nonlinear ODEs obtained from the equation of motion and constitutive equations numerically and the effects of non-Newtonian quantities on velocity and on shear stress are shown through graphs. Lokendra Kumar et al.[20] have investigated the problem of mixed convection on a moving vertical cylinder with suction in a micropolar fluid medium by using finite element method. They have presented the effects of micropolar parameter, suction parameter and velocity coefficient parameter on the velocity, micro-rotation and temperature functions graphically. Mehrdad Massoudi and Tran X. Phouc [21] studied the longitudinal and torsional oscillations of a cylinder in a second grade fluid and they presented the results graphically for the shear stresses at the wall.

To the extent of the knowledge of the authors, very few literatures are available on the flow due to oscillations of a rod in micropolar fluids. The problems mentioned in [6] and [10] are some examples in this direction. Hence, in this paper we consider the flow of micropolar fluid generated by a porous circular cylinder performing longitudinal and torsional oscillations and subjected to suction velocity at the surface. 


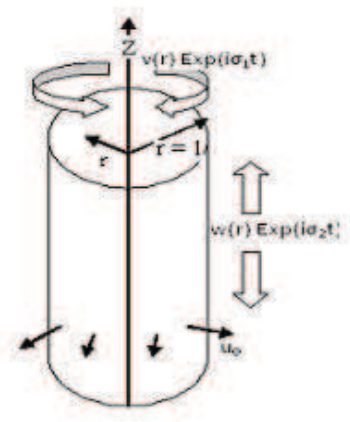

Figure 1: Geometry of the problem: non dimensional form.

\section{Formulation of the Problem}

Consider a porous circular cylinder of radius $a$ in an infinite expansion of a micropolar fluid. The cylinder is subjected to longitudinal oscillations, $\operatorname{Exp}\left(i \omega_{1} t\right)$ and torsional oscillations $\operatorname{Exp}\left(i \omega_{2} t\right)$, with amplitudes $q_{0} \sin b, q_{0} \cos b$ along the respective directions with $\omega_{1}$ as the frequency of the longitudinal oscillations, $\omega_{2}$ as the frequency of the torsional oscillations, $q_{0}$ as the magnitude of oscillations and $\mathrm{b}$ is the angle between the direction of torsional oscillation and the base vector e. i.e The cylinder oscillates with velocity as given by the expression $\mathbf{Q}_{\Gamma}=q_{0}\left(\sin b e^{i \omega_{1} t} e_{\theta}+\cos b e^{i \omega_{2} t} e_{z}\right) . u_{0}$ is a suction or injection velocity on the surface of the porous cylinder. Cylindrical polar coordinate system is considered with the Z-axis along the axis of the cylinder and origin on the axis. Let $R, \theta$ and $Z$ denote the radial, azimuthal and axial coordinates respectively of a point in the region of flow. Now we consider the flow generated in the micropolar fluid due to the oscillations of the cylinder. The physical model illustrating the problem under consideration is shown in figurel.

After neglecting body forces and body couples, the field equations governing the incompressible microplar fluid dynamics as proposed by Eringen [3] are:

$$
\begin{aligned}
\nabla_{1} \cdot Q & =0 \\
\rho\left(\frac{\partial \mathbf{Q}}{\partial \tau}+\mathbf{Q} \cdot \nabla_{1} \mathbf{Q}\right) & =-\nabla_{1} \mathbf{P}+\kappa \nabla_{1} \times \boldsymbol{l}-(\mu+\kappa) \nabla_{1} \times \nabla_{1} \times \mathbf{Q} \\
\rho j\left(\frac{\partial \boldsymbol{l}}{\partial \tau}+\mathbf{Q} \cdot \nabla_{1} \boldsymbol{l}\right) & =-2 \kappa \boldsymbol{l}+\kappa \nabla_{1} \mathbf{Q}-\gamma \nabla_{1} \times \nabla_{1} \times \boldsymbol{l}+(\alpha+\beta+\gamma) \nabla_{1}\left(\nabla_{1} \cdot \boldsymbol{l}\right)
\end{aligned}
$$

where $\mathbf{Q}$ is velocity vector, $\mathbf{P}$ is fluid pressure, $\boldsymbol{l}$ is micro-rotation vector, $\rho$ is density, $\tau$ is time, $\nabla_{1}$ is the dimensional gradient. The coefficients $(\mu, \kappa)$ and $\alpha, \beta, \gamma$ are viscosity and gyro viscosity coefficients.

By nature of the geometry and flow, the velocity and micro-rotation components are assumed 
to be axially symmetric and depend only on radial distance and time. Hence the velocity and micro-rotations are taken in the form

$$
\begin{gathered}
\mathbf{Q}=U(R) e_{r}+V(R, \tau) e_{\theta}+W(R, \tau) e_{z} \\
\boldsymbol{l}=A(R) e_{r}+B(R, \tau) e_{\theta}+C(R, \tau) e_{z}
\end{gathered}
$$

Let us introduce the following non dimensional scheme

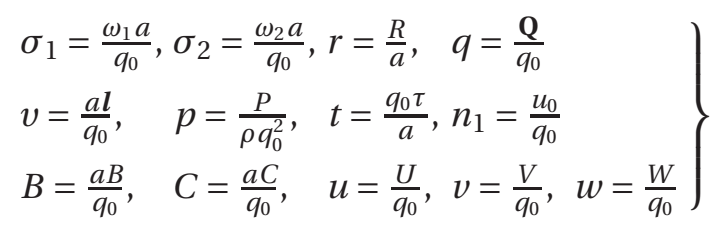

By the non-dimensional scheme (6) in (2) and (3), the equations for the flow are transformed to the following non-dimensional form

$$
\begin{aligned}
\operatorname{Re}(1-c)\left(\frac{\partial \mathbf{q}}{\partial t}+\mathbf{q} \cdot \nabla \mathbf{q}\right) & =-(1-c) \operatorname{Re} \nabla p+c \nabla \times v-\nabla \times \nabla \times \mathbf{q} \\
\varepsilon\left(\frac{\partial v}{\partial t}+\mathbf{q} \cdot \nabla v\right) & =-2 s v+s \nabla \times \mathbf{q}-\nabla \times \nabla \times v+\frac{1}{\delta} \nabla(\nabla \cdot v)
\end{aligned}
$$

where the non-dimensional parameters viz. Reynolds number Re, cross viscosity parameter or coupling number c, couple stress parameters $s$ and $\delta$ and gyration parameter $\varepsilon$ are defined by

$$
R e=\frac{\rho q_{0} a}{\mu}, c=\frac{\kappa}{\mu+\kappa}, s=\frac{\kappa a^{2}}{\gamma}, \delta=\frac{\rho j q_{0} a}{\gamma}, \delta=\frac{\gamma}{\alpha+\beta+\gamma}
$$

Now to match with the oscillating boundary, the velocity in (4) and micro-rotation in (5) are assumed in the form

$$
\mathbf{q}=u(r) e_{r}+v(r) e^{i \sigma_{1} t} e_{\theta}+w(r) e^{i \sigma_{2} t} e_{z} \quad a n d v=A(r) e_{r}+B(r) e^{i \sigma_{2} t} e_{\theta}+C(r) e^{i \sigma_{1} t} e_{z}
$$

where $\mathrm{u}=$ suction velocity in radial direction is taken as $\frac{n_{1}}{r}$ to satisfy the incompressibility condition. Here $n_{1}$ is the suction parameter defined in (6).

The equation (7) will give raise to the following three scalar equations in the directions of base vectors

$$
\begin{aligned}
\frac{n_{1}^{2}}{r^{3}}+\frac{v^{2}}{r} e^{2 i \sigma_{1} t} & =\frac{d p}{d r} \\
\operatorname{Re}(1-c)\left(i \sigma_{1} v+\frac{n_{1}}{r}\left(\frac{d v}{d r}+\frac{v}{r}\right)\right) & =-c \frac{d C}{d r}+D^{2} v \\
\operatorname{Re}(1-c)\left(i \sigma_{2} w+\frac{n_{1}}{r} \frac{d w}{d r}\right) & =c\left(\frac{d B}{d r}+\frac{B}{r}\right)+\left(\frac{d}{d r}+\frac{1}{r}\right) \frac{d w}{d r}
\end{aligned}
$$


where $D^{2}=\frac{d^{2}}{d r^{2}}+\frac{1}{r} \frac{d}{d r}-\frac{1}{r^{2}}$

Similarly the equation (8) will yield the following three equations along the base vectors

$$
\begin{aligned}
\varepsilon\left(\frac{n_{1}}{r} \frac{d A}{d r}-\frac{v B}{r} e^{i\left(\sigma_{1}+\sigma_{2}\right) t}\right) & =-2 s A+\frac{1}{\delta} \frac{d}{d r}\left(\frac{1}{r} \frac{d(r A)}{d r}\right) \\
\varepsilon\left(i \sigma_{2} B+\frac{n_{1}}{r} \frac{d B}{d r}+\frac{v A}{r} e^{i\left(\sigma_{1}-\sigma_{2}\right) t}\right) & =-2 s B-s \frac{d w}{d r}+D^{2} B \\
\varepsilon\left(i \sigma_{1} C+\frac{n_{1}}{r} \frac{d C}{d r}\right) & =-2 s C+s\left(\frac{d v}{d r}+\frac{v}{r}\right)+\left(\frac{d}{d r}+\frac{1}{r}\right) \frac{d C}{d r}
\end{aligned}
$$

Eliminating $C$ from (12) and (16), we get the following equation for velocity component $v$ as

$$
\begin{gathered}
\left(D^{2}-\varepsilon \frac{n_{1}}{r} \frac{d}{d r}+\varepsilon \frac{n_{1}}{r^{2}}-2 s-i \sigma_{1} \varepsilon\right) \\
+\left(D^{2}-\operatorname{Re}(1-c) i \sigma_{1}-\frac{\operatorname{Re}(1-c) n_{1}}{r}\left(\frac{d}{d r}+\frac{1}{r}\right)\right) v+c s D^{2} v=0
\end{gathered}
$$

We note the following relations:

$$
\left.\begin{array}{l}
D^{4} v=v^{i v}+\frac{2}{r} v^{\prime \prime \prime}-\frac{3}{r^{2}} v^{\prime \prime}+\frac{3}{r^{3}} v^{\prime}-\frac{3}{r^{4}} v \\
D^{2}\left(\frac{1}{r} v^{\prime}\right)=\frac{1}{r} v^{\prime \prime \prime}-\frac{1}{r^{2}} v^{\prime \prime} \\
D^{2}\left(\frac{1}{r^{2}} v\right)=\frac{1}{r^{2}} v^{\prime \prime}-\frac{3}{r^{3}} v^{\prime}+\frac{3}{r^{4}} v \\
\frac{1}{r} \frac{d}{d r} D^{2} v=\frac{1}{r} v^{\prime \prime \prime}+\frac{1}{r^{2}} v^{\prime \prime}-\frac{2}{r^{3}} v^{\prime}+\frac{2}{r^{4}} v
\end{array}\right\}
$$

Using these four relations (18) in the equation (17) we get

$$
v^{i v}+\frac{a_{1}}{r} v^{\prime \prime \prime}+\left(a_{2}+\frac{a_{3}}{r^{2}}\right) v^{\prime \prime}+\left(\frac{a_{4}}{r}+\frac{a_{5}}{r^{3}}\right) v^{\prime}+\left(a_{6}+\frac{a_{7}}{r^{2}}+\frac{a_{8}}{r^{4}}\right) v=0
$$

where

$$
\begin{aligned}
& a_{1}=2-\varepsilon n_{1}-\operatorname{Re}(1-c) n_{1} \\
& a_{2}=-(2-c) s-i \sigma_{1}(\varepsilon+\operatorname{Re}(1-c)) \\
& a_{3}=\varepsilon \operatorname{Re}(1-c) n_{1}^{2}-3 \\
& a_{4}=-(2-c) s+2 s \operatorname{Re}(1-c) n_{1}+i \sigma_{1}\left(2 \operatorname{Re}(1-c) n_{1} \varepsilon-\operatorname{Re}(1-c)-\varepsilon\right) \\
& a_{5}=3\left(1+\operatorname{Re}(1-c) n_{1}+\varepsilon n_{1}\right)-\varepsilon \operatorname{Ren}_{1}^{2}(1-c) \\
& a_{6}=-\sigma_{1}^{2} \operatorname{Re}(1-c) \varepsilon+2 i s \sigma_{1} \operatorname{Re}(1-c) \\
& a_{7}=(2-c) s+i \sigma_{1}(\varepsilon+\operatorname{Re}(1-c))+2 s \operatorname{Re}(1-c) n_{1} \\
& a_{8}=-3\left(1+\varepsilon n_{1}+\operatorname{Re}(1-c) n_{1}+\operatorname{Re}(1-c) n_{1}^{2} \varepsilon\right)
\end{aligned}
$$

We assume $\mathrm{A}=0$ and neglect $v B$ in the convective term of (14) in view of smallness of $v$ and $B$. Hence equation (14) is identically satisfied. Now eliminating w from the equations (13) and (15), we get the following equation for $B$ :

$$
\left(D^{2}-\frac{R e(1-c) n_{1}}{r} \frac{d}{d r}+\frac{\operatorname{Re}(1-c) n_{1}}{r^{2}}-i \sigma_{2} \operatorname{Re}(1-c)\right)
$$




$$
+\left(D^{2}-\frac{\varepsilon n_{1}}{r} \frac{d}{d r}-2 s-i \sigma_{2} \varepsilon\right) B+c s D^{2} B=0
$$

Using the relations (18) in terms of $B$ in (21) we have

$$
B^{i v}+\frac{b_{1}}{r} B^{\prime \prime \prime}+\left(b_{2}+\frac{b_{3}}{r^{2}}\right) B^{\prime \prime}+\left(\frac{b_{4}}{r}+\frac{b_{5}}{r^{3}}\right) B^{\prime}+\left(b_{6}+\frac{b_{7}}{r^{2}}+\frac{b_{8}}{r^{4}}\right) B=0
$$

where

$$
\begin{aligned}
& b_{1}=2-\varepsilon n_{1}-\operatorname{Re}(1-c) n_{1} \\
& b_{2}=-(2-c) s-i \sigma_{2}(\varepsilon+\operatorname{Re}(1-c)) \\
& b_{3}=\varepsilon n_{1}+\varepsilon \operatorname{Re}(1-c) n_{1}^{2}-3 \\
& b_{4}=-(2-c) s+2 s \operatorname{Re}(1-c) n_{1}+i \sigma_{2}\left(2 \operatorname{Re}(1-c) n_{1} \varepsilon-\operatorname{Re}(1-c)-\varepsilon\right) \\
& \left.b_{5}=3\left(1+\operatorname{Re}(1-c) n_{1}\right)+\varepsilon n_{1}\right)-2 \varepsilon \operatorname{Ren}_{1}^{2}(1-c) \\
& b_{6}=-\sigma_{2}^{2} \operatorname{Re}(1-c) \varepsilon+2 i s \sigma_{2} \operatorname{Re}(1-c) \\
& b_{7}=(2-c) s+i \sigma_{2}\left(\varepsilon+\operatorname{Re}(1-c)-\operatorname{Re}(1-c) \varepsilon n_{1}\right)-2 s \operatorname{Re}(1-c) n_{1} \\
& b_{8}=-3\left(1+\operatorname{Re}(1-c) n_{1}\right)
\end{aligned}
$$

Eliminating $\frac{d C}{d r}$ from (12) and (16) we get the equation for $C$ in terms of $v$ as below

$$
\begin{aligned}
c\left(2 s+i \sigma_{1} \varepsilon\right) C= & v^{\prime \prime \prime}+\frac{2-n_{1} \varepsilon-\operatorname{Re}(1-c) n_{1}}{r} v^{\prime \prime} \\
& +\left(c s-i \sigma_{1} \operatorname{Re}(1-c)+\frac{\operatorname{Re}(1-c) n_{1}^{2} \varepsilon-1-(\operatorname{Re}(1-c)+\varepsilon) n_{1}}{r^{2}}\right) v^{\prime} \\
& +\left(\frac{c s+i \sigma_{1} \operatorname{Re}(1-c)\left(\varepsilon n_{1}-1\right)}{r}+\frac{1+\operatorname{Re}(1-c) n_{1}+n_{1} \varepsilon+\operatorname{Re}(1-c) n_{1}^{2} \varepsilon}{r^{3}}\right) v
\end{aligned}
$$

This can be written as

$$
\begin{aligned}
c\left(2 s+i \sigma_{1} \varepsilon\right) C= & v^{\prime \prime \prime}+\frac{a_{1}}{r} v^{\prime \prime}+\left(c s-i \sigma_{1} \operatorname{Re}(1-c)+\frac{2 \operatorname{Re}(1-c) n_{1}^{2} \varepsilon-a_{5}}{3 r^{2}}\right) v^{\prime} \\
& +\left(\frac{c s+i \sigma_{1} \operatorname{Re}(1-c)\left(\varepsilon n_{1}-1\right)}{r}-\frac{a_{8}}{3 r^{3}}\right) v
\end{aligned}
$$

Similarly from (13) and (15) we have the equation for $\mathrm{w}$ as follows:

$$
\begin{aligned}
i \sigma_{2} s R e(1-c) w= & B^{\prime \prime \prime}+\frac{b_{1}}{r} B^{\prime \prime}+\left(s(c-2)-i \sigma_{2} \varepsilon+\frac{\operatorname{Re}(1-c) n_{1}^{2} \varepsilon-b_{5}}{3 r^{2}}\right) B^{\prime} \\
& +\left(\frac{i \sigma_{2} \operatorname{Re}(1-c)-b_{7}}{r}-\frac{b_{8}}{3 r^{3}}\right) B
\end{aligned}
$$

\section{Boundary conditions}

We solve the equations (19) and (22) for obtaining velocity and micro-rotation components under the following conditions. 
(1) No slip condition:

Fluid velocity on the boundary is equal to velocity of the boundary

i.e., $\mathbf{Q}_{\mathbf{R}=\mathbf{a}}=q_{0}\left(\sin b e^{i \omega_{1} t} e_{\theta}+\cos b e^{i \omega_{2} t} e_{z}\right)$

This equation in non dimensional form gives the equations

$$
v(1)=\cos b \quad \text { and } \quad w(1)=\sin b
$$

(2) Hyper stick condition

Micro-rotation vector on the boundary is equal to angular velocity of the boundary. This takes the form $v=\frac{1}{2} \nabla \times \mathbf{q}_{\Gamma}$

In scalar form this equation gives the two conditions

$$
C(1)=\sigma_{1} \quad \text { and } \quad B(1)=0
$$

As $r \rightarrow \infty$, the flow is at rest and boundary conditions can be taken as

$$
v(\infty)=0, w(\infty)=0, B(\infty)=0, C(\infty)=0
$$

Thus the equations (19) and (22) are to be solved under the eight conditions (26), (27) and (28).

\section{Finite difference method of solution}

In view of the complicated nature of two equations (19) and (22), the analytical solution for $v$ and $B$ seems to be beyond reach. The details of finite difference method used here can be studied from Ref. [22], for obtaining the solution for $v$ and $B$. We take 20 units of distance from origin is very large represents infinity. Hence we discretise the interval $[1,20]$ into $n$ subintervals with $n+1$ nodes. Each node is represented by $r_{i}=1+i h$, with $h=19 / n$ the step length, starting from first node $r_{0}=1$ to the last node $r_{n}=20$. The values of the functions $v$, $w$ at $r_{i}$ are given by $v_{i}$ and $w_{i}$. The symmetric derivative formulae at the $i^{\text {th }}$ node are given as below:

$$
\left.\begin{array}{l}
v_{i}^{\prime}=\frac{v_{i+1}-v_{i-1}}{2 h} \\
v_{i}^{\prime \prime}=\frac{v_{i+1}-2 v_{i}+v_{i-1}}{h^{2}} \\
v_{i}^{\prime \prime \prime}=\frac{v_{i+2}-2 v_{i+1}+2 v_{i-1}-v_{i-2}}{2 h^{2}} \\
v_{i}^{i v}=\frac{v_{i+2}-4 v_{i+1}+6 v_{i}-4 v_{i-1}+v_{i-2}}{h^{4}}
\end{array}\right\}
$$

Substituting these derivatives given in (29), in the equation (19) we get

$$
t_{1, i} v_{i-2}+t_{2, i} v_{i-1}+t_{3, i} v_{i}+t_{4, i} v_{i+1}+t_{5, i} v_{i+2}=0
$$


where

$$
\left.\begin{array}{l}
t_{1, i}=r_{i}^{4}-a_{1} r_{i}^{3} \frac{h}{2} \\
t_{2, i}=-4 r_{i}^{4}-a_{1} r_{i}^{3} h+\left(a_{2} r_{i}^{2}+a_{3}\right) r_{i}^{2} h^{2}-\left(a_{4} r_{i}^{3}+a_{5} r_{i}\right) \frac{h^{3}}{2} \\
t_{3, i}=6 r_{i}^{4}-2\left(a_{2} r_{i}^{4}+a_{3} r_{i}^{2}\right) h^{2}+\left(a_{6} r_{i}^{4}+a_{7} r_{i}^{2}+a_{8}\right) h^{4} \\
t_{4, i}=-4 r_{i}^{4}-a_{1} r_{i}^{3} h+\left(a_{2} r_{i}^{2}+a_{3}\right) r_{i}^{2} h^{2}+\left(a_{4} r_{i}^{3}+a_{5} r_{i}\right) \frac{h^{3}}{2} \\
t_{5, i}=r_{i}^{4}+a_{1} r_{i}^{3} \frac{h}{2}
\end{array}\right\}
$$

We take the following boundary conditions

$$
v_{0}=v(1)=\cos b=n_{2}, C_{0}=C(1)=\sigma_{1}, v_{n}=0 \text { and } C_{n}=0
$$

Evaluating (30) for different values of i we obtain

$$
\left.\begin{array}{rl}
i= & 0: t_{1,0} v_{-2}+t_{2,0} v_{-1}+t_{4,0} v_{1}+t_{5,0} v_{2}=-t_{3,0} v_{0} \\
i= & 1: t_{1,1} v_{-1}+t_{3,1} v_{1}+t_{4,1} v_{2}+t_{5,1} v_{3}=-t_{2,1} v_{0} \\
i= & 2: t_{2,2} v_{1}+t_{3,2} v_{2}+t_{4,2} v_{3}+t_{5,2} v_{4}=-t_{1,2} v_{0} \\
& \vdots \\
i= & n-1: t_{1, n-1} v_{n-3}+t_{2, n-1} v_{n-2}+t_{3, n-1} v_{n-1}+t_{5, n-1} v_{n+1}=0 \\
i= & n: t_{1, n} v_{n-2}+t_{2, n} v_{n-1}+t_{4, n} v_{n+1}+t_{5, n-1} v_{n+2}=0
\end{array}\right\}
$$

Thus the system of equations (33) represents $n+1$ equation in $n+3$ unknowns. Hence we require two more equations. These can be obtained from (24). The finite difference form of (24), will give us the equation

$$
\begin{array}{rl}
-2 h^{3} & c\left(\varepsilon i \sigma_{1}+2 s\right) C_{i}=v_{i-2} \\
& +\left(-2-\frac{2 a_{1} h}{r_{i}}+h^{2}\left(s c-\operatorname{Re}(1-c) i \sigma_{1}+\frac{2 \operatorname{Re}(1-c) n_{1}^{2} \varepsilon-a_{5}}{3 r_{i}^{2}}\right)\right) v_{i-1} \\
& +\left(\frac{4 a_{1} h}{r_{i}}-\frac{2 h^{3} a_{8}}{3 r_{i}^{3}}-\frac{2 h^{3}\left(s c-\operatorname{Rei} \sigma_{1}(1-c)\left(1-\varepsilon n_{1}\right)\right)}{r_{i}}\right) v_{i} \\
& +\left(2-\frac{2 a_{1} h}{r_{i}}-h^{2}\left(s c-\operatorname{Re}(1-c) i \sigma_{1}+\frac{2 \operatorname{Re}(1-c) n_{1}^{2} \varepsilon-a_{5}}{3 r_{i}^{2}}\right)\right) v_{i+1}-v_{i+2}
\end{array}
$$

Using the boundary conditions $C_{0}=\sigma_{1}$ and $C_{n}=0$ in (34), we get

$$
v_{-2}+t_{1} v_{-1}+t_{2} v_{1}-v_{2}=t_{3} \quad \text { and } \quad v_{n-2}+t_{4} v_{n-1}+t_{5} v_{n+1}-v_{n+2}=0
$$

where

$$
\begin{aligned}
& t_{1}=-2-\frac{2 a_{1} h}{r_{0}}+h^{2}\left(s c-i \operatorname{Re}(1-c) \sigma_{1}+\frac{2 \operatorname{Re}(1-c) n_{1}^{2} \varepsilon-a_{5}}{3 r_{0}^{2}}\right) \\
& t_{2}=2-\frac{2 a_{1} h}{r_{0}}-h^{2}\left(s c-i \operatorname{Re}(1-c) \sigma_{1}+\frac{2 \operatorname{Re}(1-c) n_{1}^{2} \varepsilon-a_{5}}{3 r_{0}^{2}}\right) \\
& t_{3}=-2 h^{3}\left(c \sigma_{1}\left(\varepsilon i \sigma_{1}+2 s\right)+\left(\frac{2 a_{1}}{r_{0} h^{2}}+\frac{\operatorname{Rei\sigma _{1}(1-c)(1-n_{1}\varepsilon )-sc}}{r_{0}}+\frac{a_{8}}{3 r_{0}^{3}}\right) n_{2}\right)
\end{aligned}
$$


$t_{4}=-2-\frac{2 a_{1} h}{r_{n}}+h^{2}\left(s c-i \operatorname{Re}(1-c) \sigma_{1}+\frac{2 \operatorname{Re}(1-c) n_{1}^{2} \varepsilon-a_{5}}{3 r_{n}^{2}}\right)$

$t_{5}=2-\frac{2 a_{1} h}{r_{n}}-h^{2}\left(s c-i \operatorname{Re}(1-c) \sigma_{1}+\frac{2 \operatorname{Re}(1-c) n_{1}^{2} \varepsilon-a_{5}}{3 r_{n}^{2}}\right)$

Expressing the equations (33)-(35) in matrix form as

$$
E X=D
$$

where

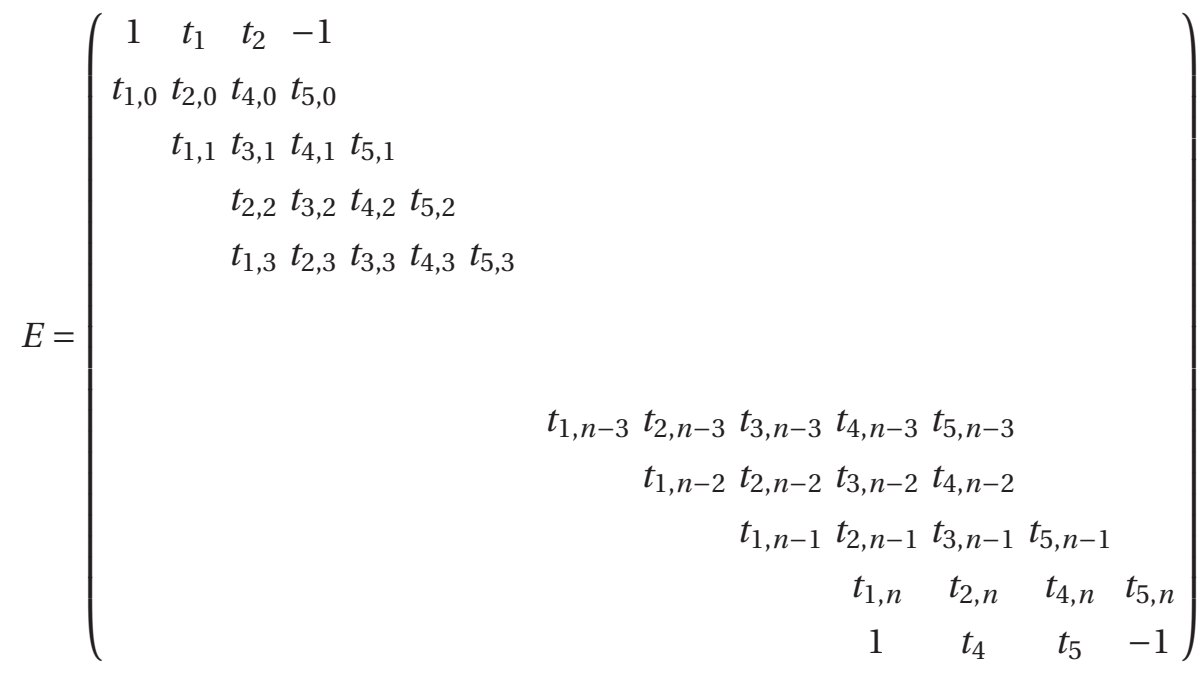

$X=\left[v_{-2}, v_{-1}, v_{1}, v_{2}, \ldots, v_{n-2}, v_{n-1}, v_{n+1}, v_{n+2}\right]^{T} \quad$ and $D=\left[t_{3},-t_{3,0} v_{0},-t_{2,1} v_{0},-t_{1,2} v_{0}, 0,0, \ldots, 0\right]^{T}$

where $v_{0}$ is the known value of $v$ on the boundary, solving the system (36) we get the solution for $v$. Hence from (34) we find $C_{i}$. Now we find solution for B and w using (22) and (25). The finite difference form of (22) is as the following:

$$
s_{1, i} B_{i-2}+s_{2, i} B_{i-1}+s_{3, i} B_{i}+s_{4, i} B_{i+1}+s_{5, i} B_{i+2}=0
$$

where

$$
\left.\begin{array}{l}
s_{1, i}=r_{i}^{4}-b_{1} r_{i}^{3} \frac{h}{2} \\
s_{2, i}=-4 r_{i}^{4}-b_{1} r_{i}^{3} h+\left(b_{2} r_{i}^{2}+b_{3}\right) r_{i}^{2} h^{2}-\left(b_{4} r_{i}^{3}+b_{5} r_{i}\right) \frac{h^{3}}{2} \\
s_{3, i}=6 r_{i}^{4}-2\left(b_{2} r_{i}^{4}+b_{3} r_{i}^{2}\right) h^{2}+\left(b_{6} r_{i}^{4}+b_{7} r_{i}^{2}+b_{8}\right) h^{4} \\
s_{4, i}=-4 r_{i}^{4}-b_{1} r_{i}^{3} h+\left(b_{2} r_{i}^{2}+b_{3}\right) r_{i}^{2} h^{2}+\left(b_{4} r_{i}^{3}+b_{5} r_{i}\right) \frac{h^{3}}{2} \\
s_{5, i}=r_{i}^{4}+b_{1} r_{i}^{3} \frac{h}{2}
\end{array}\right\}
$$

Applying the boundary conditions

$$
B_{0}=B(1)=0, w_{0}=w(1)=\sin b=n_{3}, B_{n}=0 \text { and } w_{n}=0
$$

The finite difference scheme of (25), will give us the equation

$$
-2 h^{3} i \sigma_{2} \operatorname{Re}(1-c) s w_{i}=B_{i-2}
$$




$$
\begin{aligned}
& +\left(-2-\frac{2 b_{1} h}{r_{i}}+h^{2}\left(s(c-2)-i \sigma_{2} \varepsilon+\frac{\operatorname{Re}(1-c) n_{1}^{2} \varepsilon-b_{5}}{3 r_{i}^{2}}\right)\right) B_{i-1} \\
& +\left(\frac{4 b_{1} h}{r_{i}}+\frac{2 h^{3} b_{8}}{3 r_{i}^{3}}-\frac{2 h^{3}\left(R e i \sigma_{2}(1-c)-b_{7}\right)}{r_{i}}\right) B_{i} \\
& \left(2-\frac{2 b_{1} h}{r_{i}}-h^{2}\left(s(c-2)-i \sigma_{2} \varepsilon+\frac{\operatorname{Re}(1-c) n_{1}^{2} \varepsilon-b_{5}}{3 r_{i}^{2}}\right)\right) B_{i+1}-B_{i+2}
\end{aligned}
$$

Using the boundary conditions $w_{0}=n_{3}$ and $\quad w_{n}=0$ then From (40) we have

$$
B_{-2}+s_{1} B_{-1}+s_{2} B_{1}-B_{2}=s_{3} n_{3} \quad \text { and } \quad B_{n-2}+s_{4} B_{n-1}+s_{5} B_{n+1}-B_{n+2}=0
$$

where

$$
\begin{aligned}
& s_{1}=-2-\frac{2 b_{1} h}{r_{0}}+h^{2}\left(s(c-2)-i \sigma_{2} \varepsilon+\frac{R e(1-c) n_{1}^{2} \varepsilon-b_{5}}{3 r_{0}^{2}}\right) \\
& s_{2}=2-\frac{2 b_{1} h}{r_{0}}-h^{2}\left(s(c-2)-i \sigma_{2} \varepsilon+\frac{R e(1-c) n_{1}^{2} \varepsilon-b_{5}}{3 r_{0}^{2}}\right) \\
& s_{3}=-2 h^{3} i \sigma_{2} \operatorname{Re}(1-c) s \\
& s_{4}=-2-\frac{2 b_{1} h}{r_{n}}+h^{2}\left(s(c-2)-i \sigma_{2} \varepsilon+\frac{R e(1-c) n_{1}^{2} \varepsilon-b_{5}}{3 r_{n}^{2}}\right) \\
& s_{5}=2-\frac{2 b_{1} h}{r_{n}}-h^{2}\left(s(c-2)-i \sigma_{2} \varepsilon+\frac{R e(1-c) n_{1}^{2} \varepsilon-b_{5}}{3 r_{n}^{2}}\right)
\end{aligned}
$$

From (37) substituting $i=0,1,2, \ldots, n-1, n$ and from (41), we express in matrix form as

$$
F Y=G
$$

where

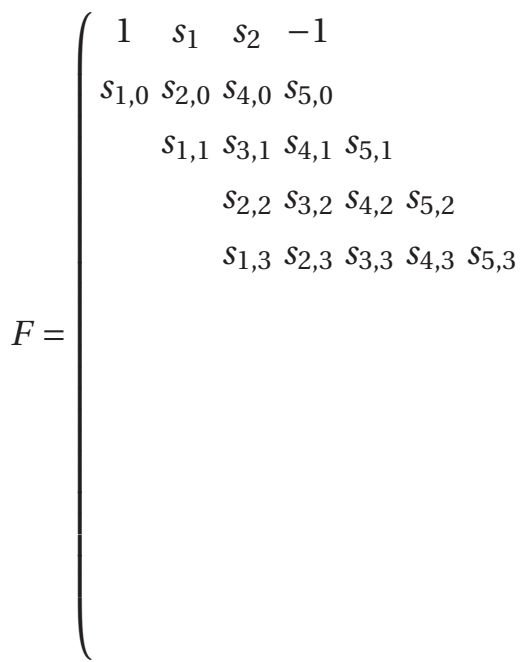$$
\left.\begin{array}{rccccc}
s_{1, n-3} s_{2, n-3} & s_{3, n-3} & s_{4, n-3} & s_{5, n-3} & & \\
s_{1, n-2} & s_{2, n-2} & s_{3, n-2} & s_{4, n-2} & & \\
s_{1, n-1} & s_{2, n-1} & s_{3, n-1} & s_{5, n-1} & \\
s_{1, n} & s_{2, n} & s_{4, n} & s_{5, n} \\
1 & s_{4} & s_{5} & -1
\end{array}\right)
$$

$Y=\left[B_{-2}, B_{-1}, B_{1}, B_{2}, \ldots, B_{n-2}, B_{n-1}, B_{n+1}, B_{n+2}\right]^{T}$ and $G=\left[s_{3} n_{3}, 0,0,0,0, \ldots, 0,0\right]^{T}$

where $w_{0}$ is the known value of $\mathrm{w}$ on the boundary. Solving the system (40) we get the solution 


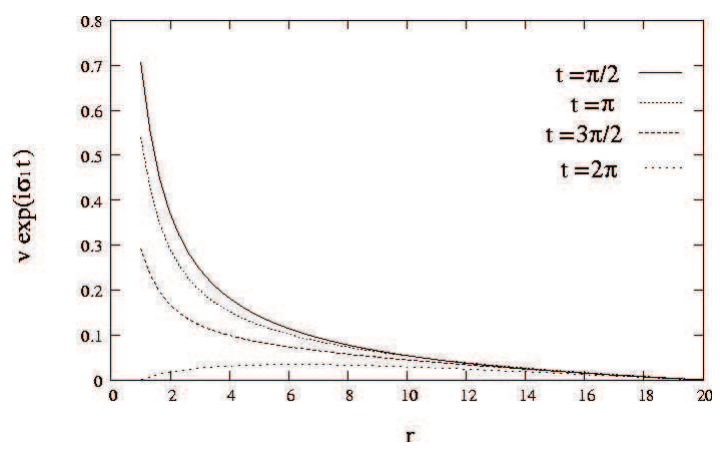

Figure 2: Variation of $v e^{i \sigma_{1} t}$ with $t$.

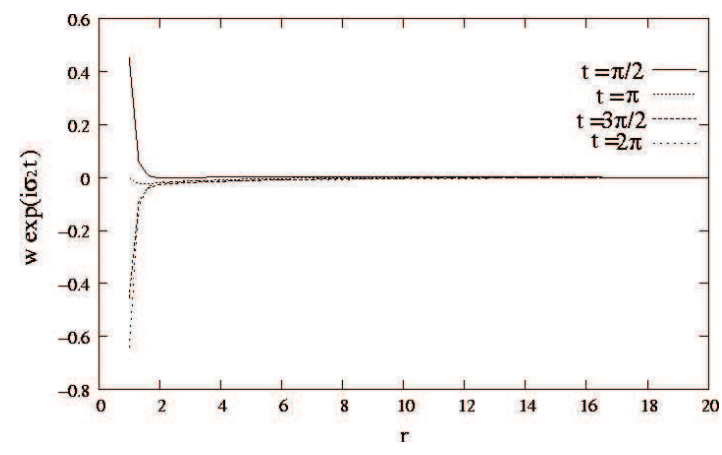

Figure 3: Variation of $w e^{i \sigma_{2} t}$ with $t$.

for $w_{i}$.

The effects of $c, s, R e, \varepsilon$ on velocity and micro-rotation components are shown graphically.

\section{Drag on the cylinder}

The drag $D$ acting on the cylinder of length $L$ is given by,

$$
D=a L \int_{0}^{2 \pi}\left(T_{21} \cos b+T_{31} \sin b\right) d \theta=2 a L \pi\left(T_{21} \cos b+T_{31} \sin b\right)_{r=1}
$$

The constitutive equation for stress tensor $T_{i j}$ of a micropolar fluid is given by

$$
T_{i j}=(-P+\lambda \nabla \cdot \mathbf{Q}) \delta_{i j}+(2 \mu+\kappa) e_{i j}+\kappa \varepsilon_{i j m}\left(\omega_{m}-\boldsymbol{l}_{m}\right)
$$

where $\omega$ is the vorticity vector, $e_{i} j$ is strain rate tensor, $\delta_{i j}$ is Kronecker delta and $\varepsilon_{i j m}$ is the alternating symbol.

The stress components given in the equation (43), are obtained from (44) in non-dimensional as

$$
\begin{gathered}
\overline{T_{21}}=\left(\frac{v_{1}-v_{-1}}{2 h}-\frac{v_{0}-c \sigma_{1}}{1-c}\right) \operatorname{Exp}\left(i \sigma_{1} t\right) \\
\overline{T_{31}}=\left(\frac{w_{1}-w_{-1}}{2 h}-\frac{c B_{0}}{1-c}\right) \operatorname{Exp}\left(i \sigma_{2} t\right)
\end{gathered}
$$

Substituting (45) and (46) in (43) we get the non-dimensional drag acting on the cylinder is given by

$$
D^{\prime}=\left(\frac{v_{1}-v_{-1}}{2 h}-\frac{v_{0}-c \sigma_{1}}{1-c}\right) \cos b \operatorname{Exp}\left(i \sigma_{1} t\right)+\left(\frac{w_{1}-w_{-1}}{2 h}-\frac{c B_{0}}{1-c}\right) \sin b \operatorname{Exp}\left(i \sigma_{2} t\right)
$$

The non-dimensional drag $D^{\prime}$ is numerically evaluated and plotted in the form of graphs for different parameters. 


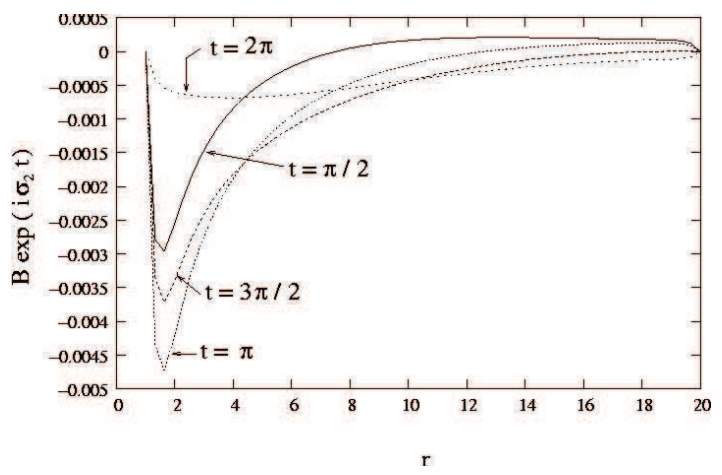

Figure 4: Variation of $B e^{i \sigma_{2} t}$ with $t$.

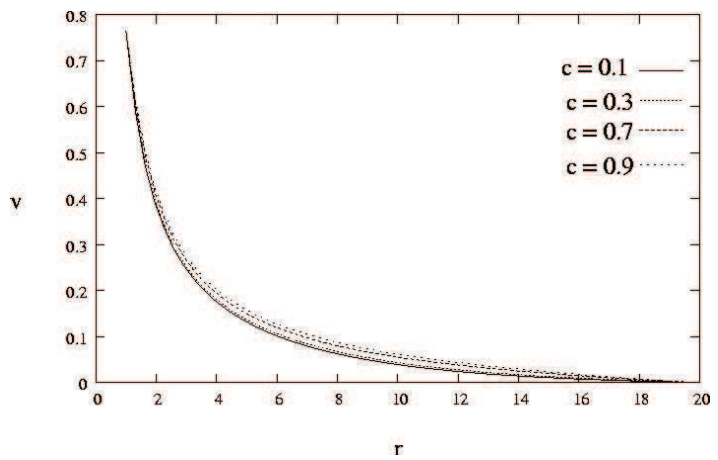

Figure 6: Variation of $v$ with $c$.

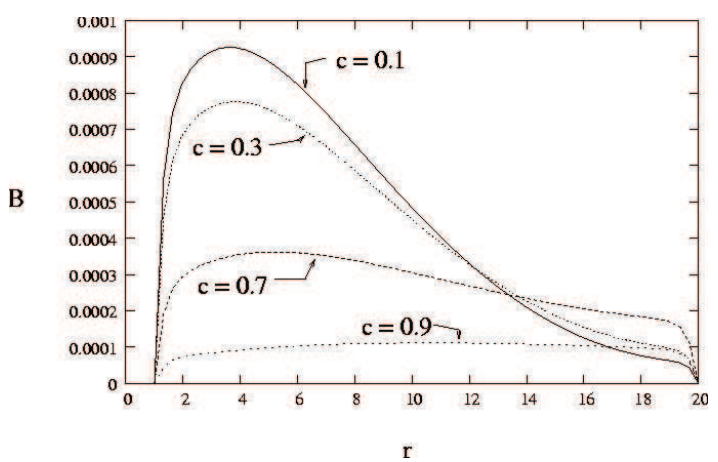

Figure 8: Variation of $B$ with $c$.

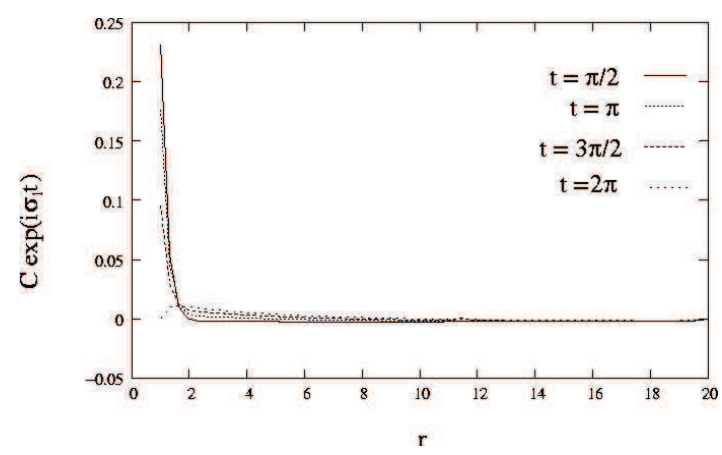

Figure 5: Variation of $C e^{i \sigma_{1} t}$ with $t$.

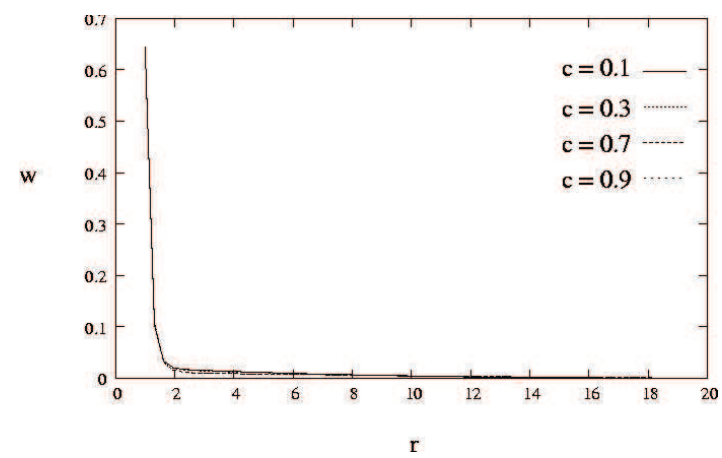

Figure 7: Variation of $w$ with $c$.

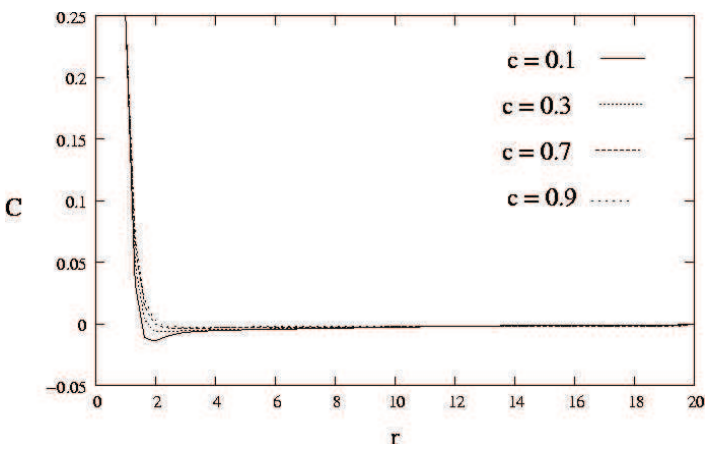

Figure 9: Variation of $C$ with $c$.

\section{Results and discussions}

The analytical expressions for the non-dimensional velocity components $v, w$, and microrotation components $B, C$ and drag are given by the equations (19), (25), (22), (24) and (47) respectively. These values depend on the values of $b$, if $b=0$, we get only torsional oscillations and if $b=\frac{\pi}{2}$, we get only axial (Longitudinal) oscillations. 


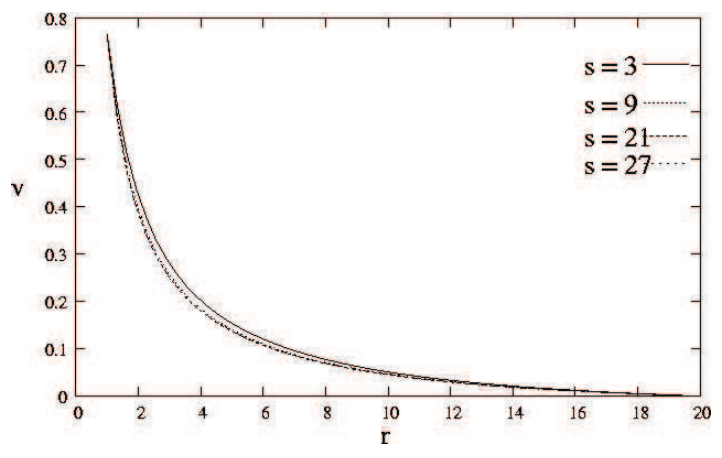

Figure 10: Variation of $v$ with $s$.

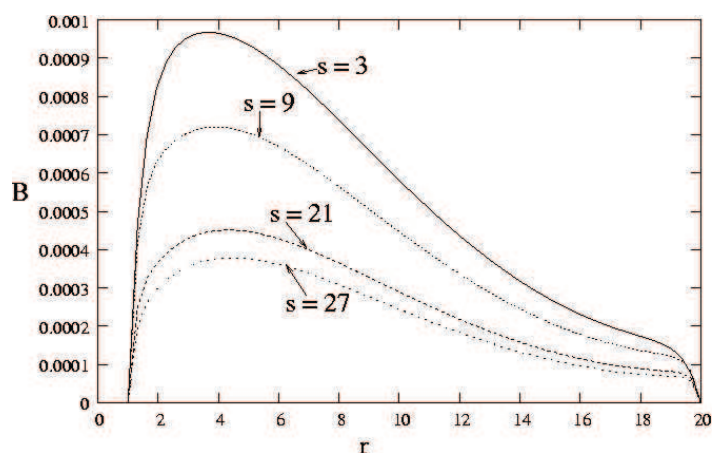

Figure 12: Variation of $B$ with $s$.

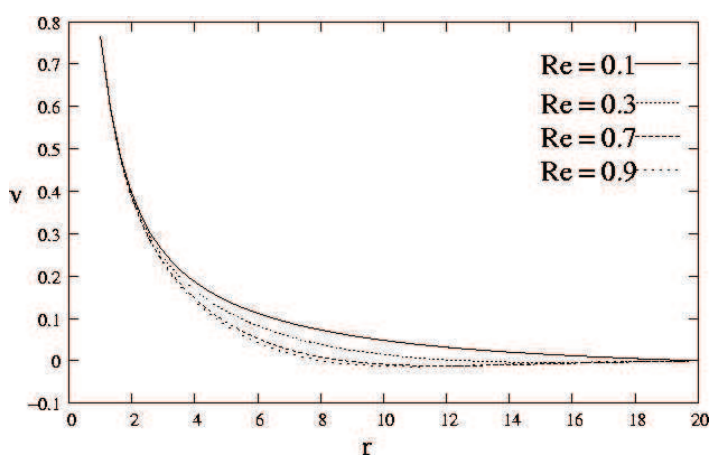

Figure 14: Variation of $v$ with $R e$.

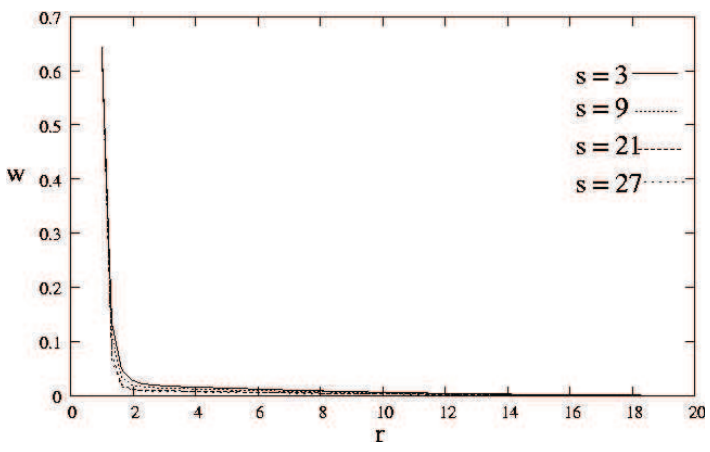

Figure 11: Variation of $w$ with $s$.

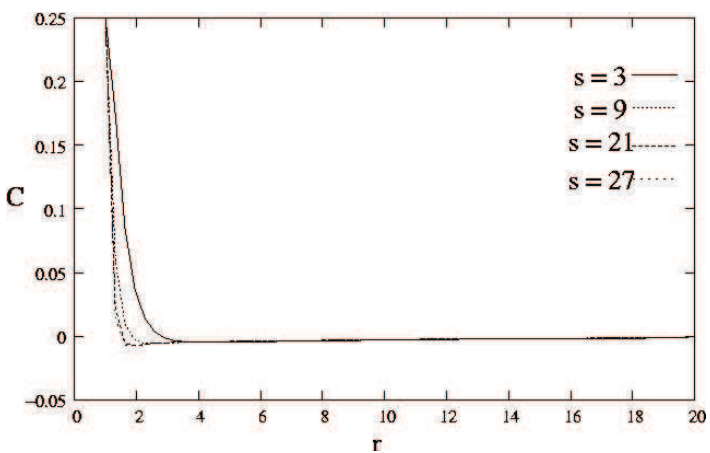

Figure 13: Variation of $C$ with $s$.

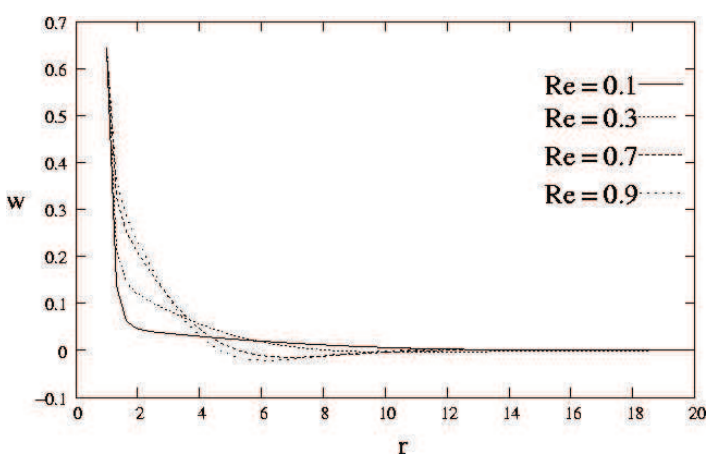

Figure 15: Variation of $w$ with $R e$.

The numerical results are presented in the form of graphs for $s=10, c=0.4, \varepsilon=0.2$, $R e=0.1, \sigma_{1}=0.25, \sigma_{2}=0.5, b=0.7, n_{1}=0.6, t=\pi$. The velocities $v$ and $w$ and microrotation components $B$ and $C$ at different non-dimensional times are shown in Figs 2-5. We notice that values of $w$ and $B$ are of the same order and $v, C$ are of the same order and values of $w$ or $B$ are $10^{-2}$ to $10^{-3}$ times less than the values of $v$ or $C$. From Figs.6-9, we notice that as the cross viscosity parameter $c$ increases, the velocities $v$ and $C$ increases, $B$ decreases and 


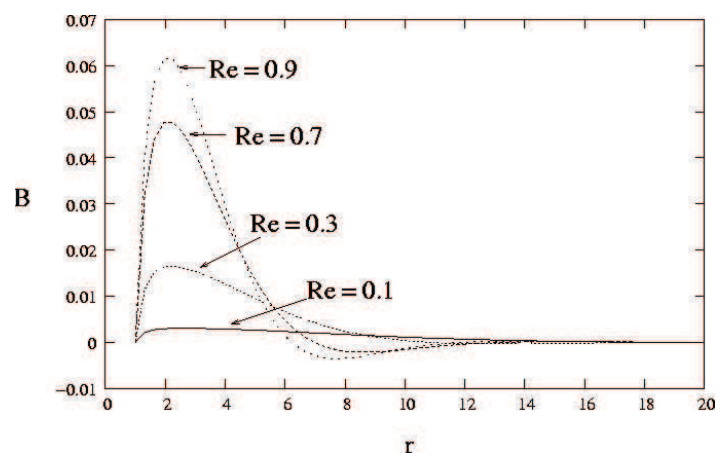

Figure 16: Variation of $B$ with $R e$.

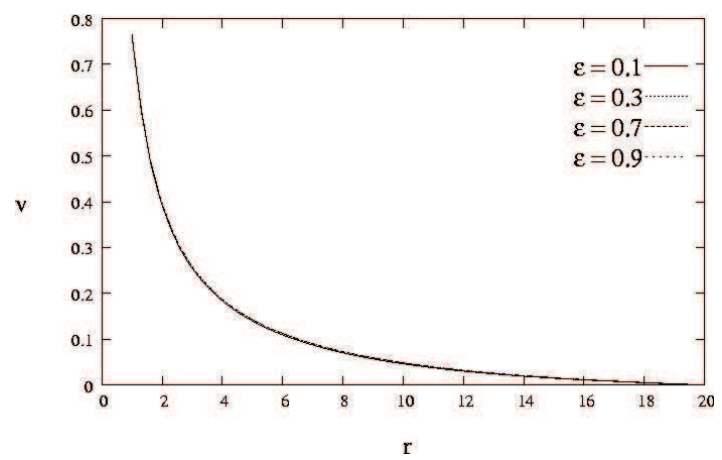

Figure 18: Variation of $v$ with $\varepsilon$.

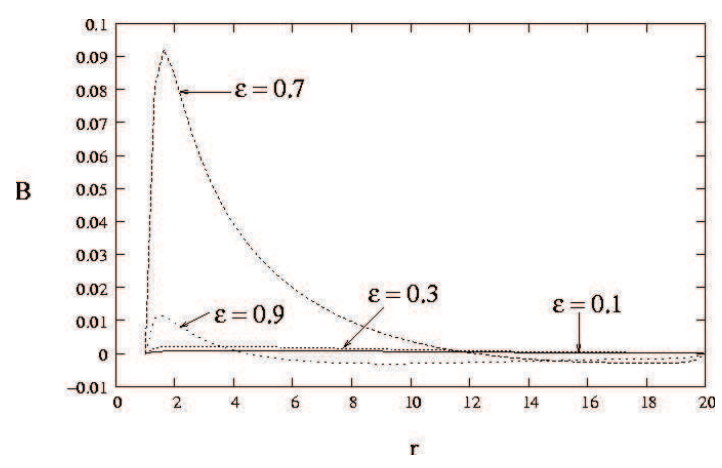

Figure 20: Variation of $B$ with $\varepsilon$.

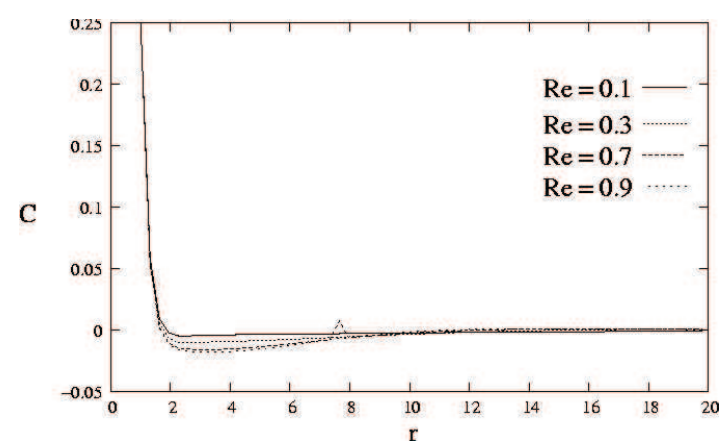

Figure 17: Variation of $C$ with $R e$.

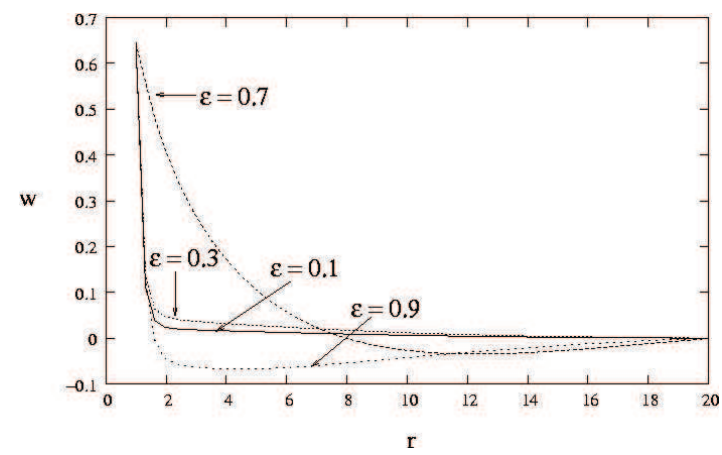

Figure 19: Variation of $w$ with $\varepsilon$.

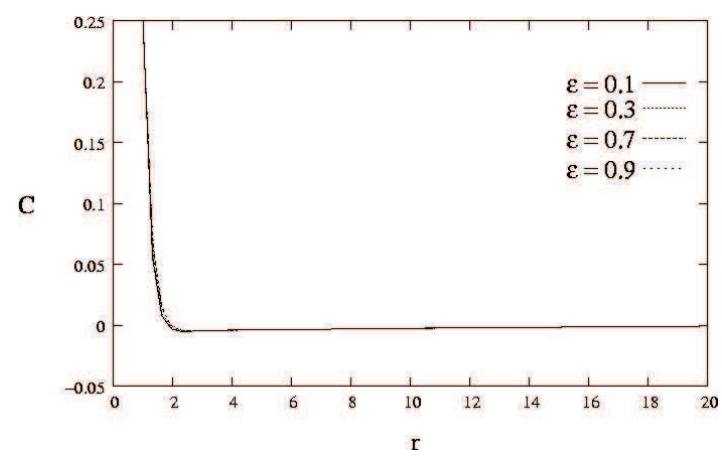

Figure 21: Variation of $C$ with $\varepsilon$.

minimum values of $v, B$ and $C$ are shifts towards origin. The axial velocity w is not affected by micropolarity parameter or cross viscosity parameter $c$. From Figs 10-13, we see that as the couple stress parameter $s$ increases, both the velocity and micro-rotation components decreases. From Figs 14-17 as the Reynolds number Re increases, the values of $v$ and $C$ decreases whereas w and B increases. In the Figs.18-21, as the Gyration parameter $\varepsilon$ increases the velocities $\mathrm{w}$ and micro-rotation components $\mathrm{B}$ are increasing. But the effect of $\varepsilon$ on the 


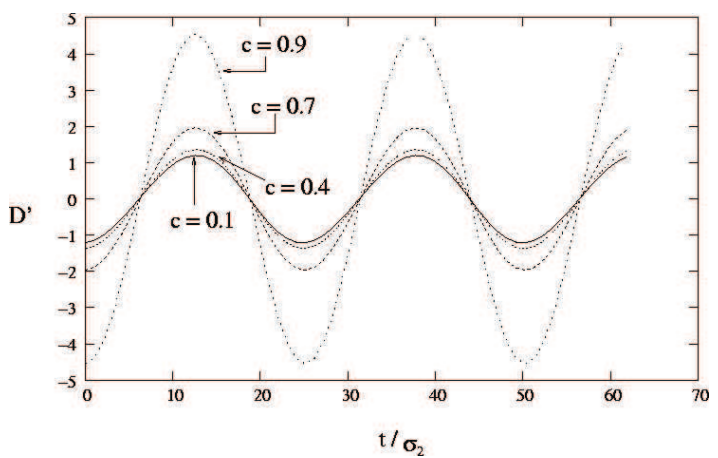

Figure 22: Variation of $D^{\prime}$ with $c$.

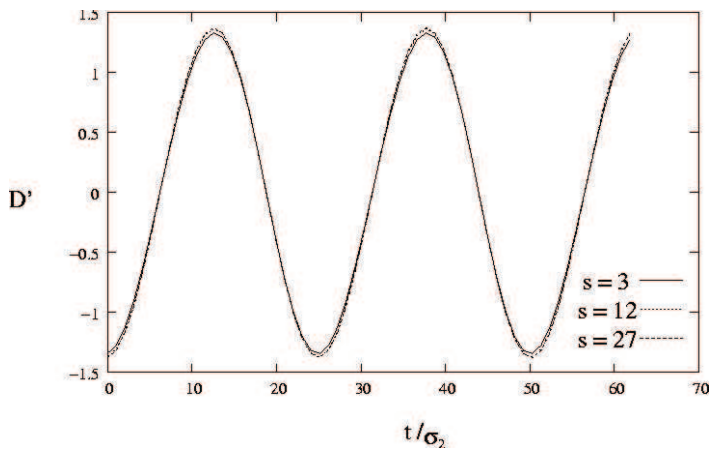

Figure 24: Variation of $D^{\prime}$ with $s$.

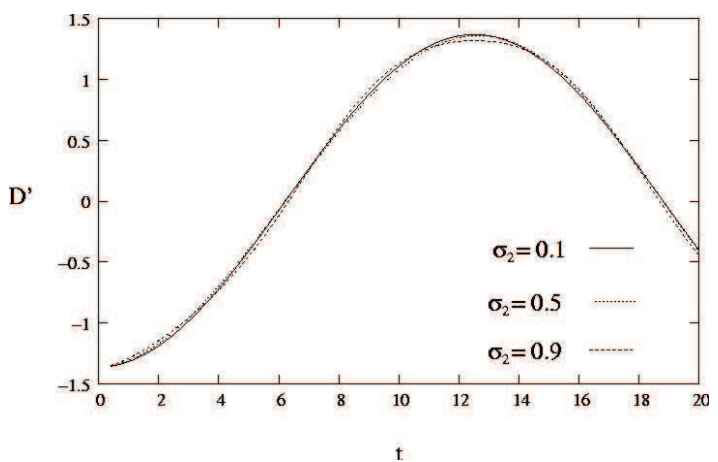

Figure 26: Variation of $D^{\prime}$ with $\sigma_{2}$.

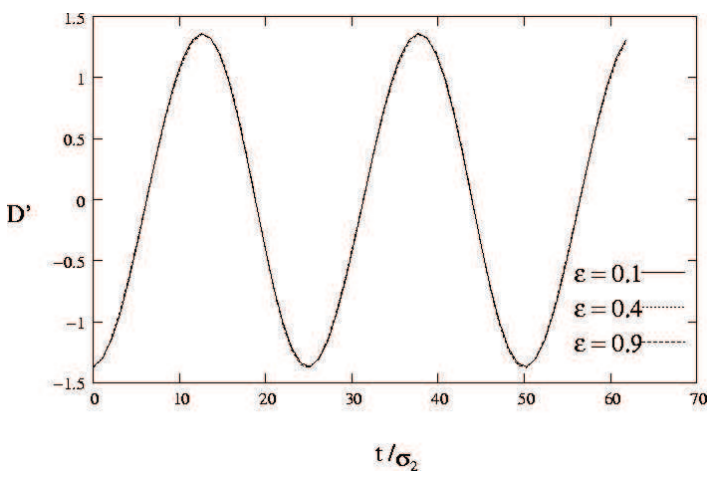

Figure 23: Variation of $D^{\prime}$ with $\varepsilon$.

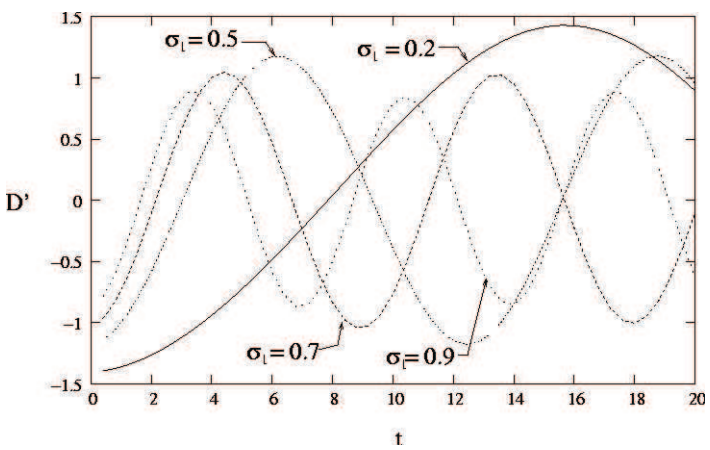

Figure 25: Variation of $D^{\prime}$ with $\sigma_{1}$.

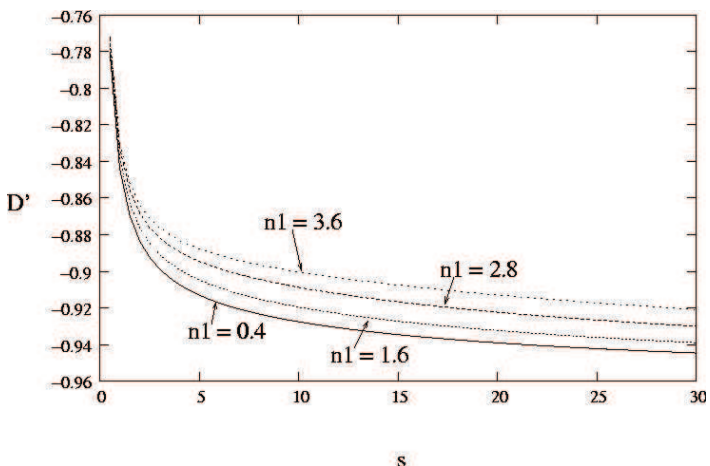

Figure 27: Variation of $D^{\prime}$ with $n_{1}$.

values of $v$ and $C$ is not very significant. i.e., the variation in the values of $\varepsilon$ does not result in much variation in the values of $v$ and $C$. From the figures of velocities for $v$ and $w$, it is observed that the axial or vertical velocity comes to zero very near to the cylinder where as the toroidal or torsional velocity $v$ dies far from the cylinder. Hence the derivatives of $w$ are large near the cylinder in comparison with the derivatives of $v$. Hence drag is more affected 


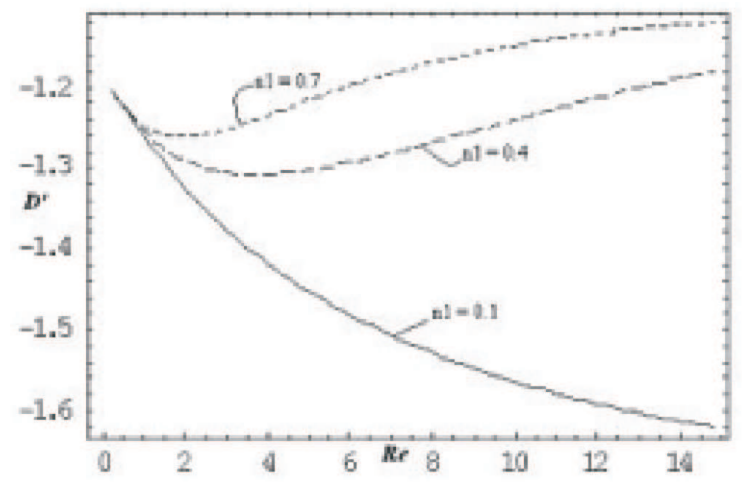

Figure 28: Variation of $D^{\prime}$ with $n_{1}$.

by axial velocity $w$.

The non-dimensional drag is calculated numerically for different values non-dimensional time in multiples of $\frac{\pi}{\sigma_{1}}$ at fixed values of $\sigma_{1}, \sigma_{2}$ and the results are shown in the Figs.22-24, In the equations of motion, local acceleration term dominates if $\sigma_{1}, \sigma_{2}$ are large. To have all terms of LHS of the same order in equations (12), (13), (15) and (16), the frequency parameters $\sigma_{1}, \sigma_{2}$ are to be small. Hence we take $\left|\sigma_{1}\right|,\left|\sigma_{2}\right|<1$. In the calculation of drag also we observe that if $\left|\sigma_{1}\right|<1,\left|\sigma_{2}\right|<1$, the drag will be within reasonable values. It can be seen from Fig.22 that as $c$ increases, the amplitude of oscillation for drag increases to high values. From fig.23 and fig 24, it is observed that couple stress parameter $s$ and gyration parameter $\varepsilon$ have very little effect on drag, when frequency parameter $\sigma_{2}$ is fixed. In figs $25-26$, we notice that as frequency parameter $\sigma_{1}$ increase drag decreases and frequency of oscillation also decreases and drag is not much affected by the parameter $\sigma_{2}$. From fig 27 as couple stress parameter $s$ increases drag increases numerically for small values of $s<5$ and for large values of $s$ drag is almost constant. From fig 28, we note that as Reynolds number increases drag increases numerically for small values of suction; but for higher values of suction rate as Reynolds number increases, drag remains almost constant. From fig 27 and 28., we note that as suction rate $n_{1}$ increases drag decreases numerically.

\section{Conclusions}

In this paper we observe that,

(1) The effect of couple stress parameter $s$ on the flow is insignificant. i.e. with respect to $s$, the velocities of viscous fluid and micropolar fluids are almost same and hence drag is not effected by couple stresses

(2) The drag offered by viscous fluids is less than that of micropolar fluids

(3) suction on the cylinder decreases the drag. 


\section{References}

[1] A. C. Eringen, Simple microfluids, Int. J. Engng. Sci., 2(1964), 205-217.

[2] A. C. Eringen, Proceedings of XI'th International Congress of Applied Mechanics, Springer-Verlag, Berlin (1965), 131.

[3] A. C. Eringen, Theory of micropolar fluids, J. Math. Mech, 16(1966), 1-18.

[4] T. Ariman, M. A. Turk and N. D. Sylvester, Microcontinuum fluid mechanics-a review, Int. J. Engng. Sci., 11(1973), 905-930.

[5] T. Ariman, M. A. Turk and N. D. Sylvester, Applications of microcontinuum fluid mechanics, Int. J. Engng. Sci, 12(1974), 273-293.

[6] C. Calmelet-Eluhu and D. R. Mazumdar, Flow of Micropolar fluid through a circular cylinder subject to longitudinal and torsinal oscillations, Mathl. Comput. Modelling, 27(1998), 69-78.

[7] G. G. Stokes, On the effect of rotation of cylinders and spheres about their own axes in increasing the logarithmic decrement of the arc of vibration, (Mathematical and Philosophical Papers 5) Cambridge: Cambridge University Press, England(1886), 207-214.

[8] M. J. Casarella and P. A. Laura, Drag on oscillating rod with longitudinal and torsional Motion, J. Hydronaut, 3(1969), 180-183.

[9] K. R. Rajagopal, Longitudinal and Torsional oscillation of a rod in a non-Newtonian fluid, Acta. Mech., 49(1983), 281-285.

[10] H. Ramkissoon and S. R. Majumdar, Flow due to the longitudinal and torsinal oscillations of a cylinder, ZAMP, 41(1990), 598-603.

[11] H. Ramkissoon, C. V. Easwaran and S. R. Majumdar, Longitudinal and Torsional oscillation of a rod in a polar fluid, Int. J. Engng. Sci., 29(1991), 215-221.

[12] D. Owen and K. Rahaman, On the flow of an Oldroyd-B liquid through a straight circular tube performing longitudinal and torsional oscillations of different frequencies, Mathematica, 14(2006), 1-9.

[13] A. S. Berman, Laminar flow in channels with porous walls, J. Appl. Phys., 24(1953), 1232-1235.

[14] J. N. Kapur, Flow of a non-Newtonian fluid between rotating cylinders with suction and injection, Phys. Fluids, 6(1963), 626-631.

[15] Jangi Lal, Unsteady flow and Heat Transfer in a viscous incompressible fluid due to the oscillations of a porous cylinder with suction, Def. Sci. J., 20(1970), 45-52.

[16] G. Pontrelli, The axisymmetric flow of a homogeneous Oldroyd-B fluid due to the longitudinal and torsional oscillations of an infinite rod, Acta. Mech., 123(1997), 57-68.

[17] M. Emin Erdogan and C. Erdem Imrak, Steady Flow of a Second-Grade Fluid in an annulus with Porous Walls, Mathematical Problems in Engineering, 2008(2008), 1-11.

[18] P. M. Rao, K. Kuwahara and K. Tsuboi, Study of unsteady viscous flow around a transversely and longitudinally oscillating circular cylinder in a uniform flow at high Reynolds number, Comput. Mech., 10(1992), 414-428.

[19] G. Pontrelli and R. K. Bhatnagar, Flow of a viscoelastic fluid between two rotating circular cylinders subject to suction or injection, Int. J. for Numer. Methods in Fluids, 24(1997), 337-349.

[20] Lokendra Kumar, Rama Bhargava and H. S. Takhar, Finite element solution of mixed convection on a moving vertical cylinder with suction in a moving micropolar fluid medium, Meccanica, 41 (2006), 63-78.

[21] Mehrdad Massoudi and Tran X. Phouc, On the motion of a second grade fluid due to longitudinal and torsional oscillations of a cylinder-A numerical study, Appl. Math. Comput, 203(2008), 471-481.

[22] P. Muthu, B. V. Rathish Kumar and Peeyush Chandra, Peristaltic motion of micropolar fluid in cylindrical tubes: effect of wall properties, Appl. Math. Modelling, 32(2008), 2019-2033.

Department of Mathematics, NIT Warangal, Warangal - 506004, India.

E-mail: jvrjosyula@yahoo.co.in

Department of Mathematics, Gitam University, Hyderabad campus, Medak-502329, India.

E-mail: naganitw@gmail.com

Department of Mathematics, NIT Warangal, Warangal - 506004, India.

E-mail: snklpm@rediffmail.com 